\begin{tabular}{|l|l|}
\hline Citation/Reference & $\begin{array}{l}\text { Lien Decruy, Jonas Vanthornhout, Tom Francart } \\
\text { Top-down modulation of neural envelope tracking: the } \\
\text { interplay with behavioral, self-report and neural measures of } \\
\text { listening effort } \\
\text { European Journal of Neuroscience 11 April 2020 }\end{array}$ \\
Archived version & $\begin{array}{l}\text { Author manuscript: the content is identical to the content of the } \\
\text { published paper, but without the final typesetting by the publisher }\end{array}$ \\
Published version & $\begin{array}{l}\text { Lien Decruy et al 2020 European Journal of Neuroscience } \\
\text { https://doi.org/10.1111/ejn.14753 } \\
\text { DoI }\end{array}$ \\
Postprint version & $\begin{array}{l}\text { https://doi.org/10.1101/815365 } \\
\text { European Journal of Neuroscience }\end{array}$ \\
\hline Author contact & $\begin{array}{l}\text { londecruy@gmail.com, jonas.vanthornhout@kuleuven.be, } \\
\text { tom.francart@kuleuven.be }\end{array}$ \\
\hline
\end{tabular}




\section{Top-down modulation of neural envelope tracking: the interplay with behavioral, self-report and neural mea- sures of listening effort}

Lien Decruy*, Damien Lesenfants, Jonas Vanthornhout, Tom Francart

${ }^{*}$ Corresponding author: Lien Decruy, KU Leuven, Department of Neurosciences, ExpORL, Herestraat 49 bus 721, B-3000 Leuven, Belgium. Telephone: +3216379840 Email: liendecruy@ gmail.com

Email adresses:

- Lien Decruy: liendecruy@gmail.com

- Damien Lesenfants: damien.lesenfants@kuleuven.be

- Jonas Vanthornhout: jonas.vanthornhout@kuleuven.be

- Tom Francart: tom.francart@med.kuleuven.be

Total number of

- pages: 28

- figures: 5

- tables: 3

- equations: 10

- words in abstract: 250

- words in text: 9222

Running title: Listening effort and neural envelope tracking

Keywords: listening effort, neural tracking of the speech envelope, EEG, speech 


\section{Abstract}

When listening to natural speech, our brain activity tracks the slow amplitude modulations of speech, also called the speech envelope. Moreover, recent research has demonstrated that this neural envelope tracking can be affected by top-down processes. The present study was designed to examine if neural envelope tracking is modulated by the effort that a person expends during listening. Five measures were included to quantify listening effort: two behavioral measures based on a novel dual-task paradigm, a self-report effort measure and two neural measures related to phase synchronization and alpha power. Electroencephalography responses to sentences, presented at a wide range of subject-specific signal-to-noise ratios, were recorded in thirteen young, normal-hearing adults. A comparison of the five measures revealed different effects of listening effort as a function of speech understanding. Reaction times on the primary task and self-reported effort decreased with increasing speech understanding. In contrast, reaction times on the secondary task and alpha power showed a peak-shaped behavior with highest effort at intermediate speech understanding levels. With regard to neural envelope tracking, we found that the reaction times on the secondary task and selfreported effort explained a small part of the variability in theta-band envelope tracking. Speech understanding was found to strongly modulate neural envelope tracking. More specifically, our results demonstrated a robust increase in envelope tracking with increasing speech understanding. The present study provides new insights in the relations among different effort measures and highlights the potential of neural envelope tracking to objectively measure speech understanding in young, normal-hearing adults. 


\section{Introduction}

Recent advances in signal processing have enabled the measurement of neural tracking of the low amplitude modulations of natural speech (Lalor \& Foxe, 2010; Ding \& Simon, 2014). Since these envelope modulations are essential for speech understanding (Shannon et al., 1995), it has been suggested to measure neural tracking of the speech envelope to assess speech understanding. Recent studies have shown an increase in envelope tracking with increasing speech understanding supporting its potential as an objective measure of speech understanding (Ding \& Simon, 2012; Vanthornhout et al., 2018; Decruy et al., 2019; Etard \& Reichenbach, 2019; Lesenfants et al., 2019). Nevertheless, it is important to take into account that top-down processes can influence neural envelope tracking. For example, there is evidence that prior knowledge affects envelope tracking. More specifically, Cervantes Constantino \& Simon (2018) found that envelope tracking persists when removing segments of the speech. Di Liberto et al. (2018) showed enhanced envelope tracking when vocoded speech was made intelligible by first presenting the unprocessed speech. Research investigating the cocktail party problem has also demonstrated a substantial influence of attention. Neural envelope tracking of the attended, target speech was found to be enhanced compared to the ignored, competing speech (Ding \& Simon, 2012; O'Sullivan et al., 2015; Das et al., 2016). In view of this, the question arises if the top-down process listening effort, defined as the deliberate allocation of mental resources to overcome obstacles in goal pursuit when carrying out a listening task (Pichora-Fuller et al., 2016), also modulates envelope tracking.

For several reasons there has been a large interest in the concept of listening effort. Firstly, it is common that two individuals achieve a similar performance on a clinical speech-in-noise test but experience a different degree of effortful listening in daily life situations (Anderson Gosselin \& Gagné, 2010). Secondly, research in which performance is equalized for two groups shows that older normal-hearing $(\mathrm{NH})$ participants expend significantly more effort during a speech-in-noise test than young NH adults (Anderson Gosselin \& Gagné, 2011). To overcome these problems, new speech-in-noise tests could be developed that require an equal amount of effort. Current, more feasible approaches involve the inclusion of listening effort measures that provide additional information to current speech-in-noise tests. We have to note, however, that the concept of listening effort is still widely debated partly due to the existence of many different methods to measure it. In the next sections, a short literature overview is given.

To measure listening effort during a behavioral experiment, a single-task or a dual-task paradigm can be used. Single-task paradigms usually involve a speech-in-noise test in which the reaction time to the recalling of heard sentences, is registered (Gatehouse \& Gordon, 
1990; Houben et al., 2013; Pals et al., 2015). A dual-task paradigm consists of two tasks and is based on the assumption that a person has a limited amount of cognitive resources that can be allocated to the two tasks (Kahneman, 1973). If the primary task is difficult, a person will allocate all their resources to this task, resulting in a limited amount of resources remaining for the secondary task. Accordingly, the performance on this secondary task represents the effort expended on the primary task. Although dual-task paradigms are widely used, no consensus is yet achieved about which type of dual-task should be used (Gagné et al., 2017). Next to behavioral tasks, self-report measures are often used to investigate listening effort since these can be easily implemented in an existing speech-in-noise test (Luts et al., 2010; Zekveld et al., 2010; Rudner et al., 2012; Wendt et al., 2014). In a study of Picou et al. (2011), participants were instructed to indicate their degree of perceived effort using a visual analog scale from 0 (no effort) to 10 (lots of effort). Although self-report measures are easily acquired, the outcome relies on the participant's ability to reliably estimate his/her listening effort as well as the participant's interpretation of the question.

To avoid this, physiological measures could be used since these do not require the cooperation of the participant. For example, Bernarding et al. (2014) proposed the phase synchronization of electroencephalography (EEG) responses to sentences as an objective measure of listening effort. More specifically, they found that less effortful conditions are reflected in the EEG by a uniform phase distribution whereas demanding conditions are associated with a clustered, non-uniform phase distribution (Bernarding et al., 2013, 2014). Next to phase, other EEG measures have also been associated with listening effort, e.g. alpha oscillations (Obleser et al., 2012; Miles et al., 2017; Dimitrijevic et al., 2019) or amplitude of event-related potentials (Bertoli \& Bodmer, 2016). Lastly, several studies have also shown that the pupil size and eye movements can be used as physiological indicators of processing load (Zekveld et al., 2010, 2018; Koelewijn et al., 2014; Wendt et al., 2014). In spite of this, the neural mechanisms underlying the changes in pupil size are complex, resulting in different outcomes compared to other effort measures (Zekveld et al., 2018; Alhanbali et al., 2019). In addition, pupillometry is affected by several factors such as age, ambient light, and requires expensive equipment as well as specialized training (Houben et al., 2013; Winn et al., 2018). These factors make it difficult to implement measures of pupil size in the clinic or research because, in contrast to EEG, pupillometry is not widely available yet.

Because listening effort can be measured using different methods, researchers often use a combination in their studies. For example, Wu et al. (2016) measured listening effort using two dual-tasks as well as a rating scale. They found that participants performed better on the easy secondary task than the hard secondary task, indicating that less effort was needed to complete the easy task. In contrast, the ratings demonstrated higher effort for the easy 
versus hard task. Different measures can thus lead to different outcomes and interpretations. Recent reviews have addressed this issue and suggest that different measures tap into different aspects of listening effort (Lemke \& Besser, 2016; Pichora-Fuller et al., 2016; Ohlenforst, Zekveld, \& Jansma et al., 2017; Alhanbali et al., 2019). For this reason, we chose to include five measures in this study. The first two measures involve a dual-task paradigm in which we register (1) the reaction time to the recalling of heard sentences and (2) the reaction time to a secondary task. According to previous research (Picou \& Ricketts, 2014) forcing competition between the same pool of resources creates a dual-task paradigm that is more sensitive to changes in listening effort. Hence, we created a new secondary task in which we ensured that the primary and secondary task required the allocation of similar cognitive resources. We chose to create a verbal working memory task as it plays an important role in speech perception (Rönnberg et al., 2013; Peelle, 2018). In addition to the dual-task, we included a (3) self-report measure in order to measure a different aspect of listening effort, perceived effort (Lemke \& Besser, 2016). For two reasons, we decided to also measure the (4) phase synchronization and (5) the alpha power of neural responses to sentences. Firstly, studies have shown an association between changes in these measures and changes in listening effort (alpha: Obleser et al., 2012; phase: Bernarding et al., 2014). Secondly, phase synchronization and alpha power are, in contrast to the previous effort measures, direct measures of brain activity similar to neural envelope tracking (Peelle, 2018; Dimitrijevic et al., 2019).

In the present study, our first aim was to compare the five listening effort measures as a function of speech understanding. All measures were evaluated across a wide range of signal-to-noise ratios (SNRs) because recent dual-task and pupillometry studies have shown a non-linear, peak-shaped behavior of listening effort as a function of SNR (Zekveld et al., 2014; Wu et al., 2016; Ohlenforst, Zekveld, \& Lunner et al., 2017). More specifically, it was shown that low SNRs not always resulted in extreme effort, but can also be associated with minimal effort when participants give up (Pichora-Fuller et al., 2016; Peelle, 2018). By evaluating each measure across a wide range of subject-specific SNRs, we extend the current understanding of listening effort measured by reaction times to heard sentences, phase synchronization and alpha power. For the behavioral and neural effort measures, we expect similar behavior because it is suggested that these measures reflect the amount of cognitive resources allocated to a task, also called processing load. Previous studies have, however, not always reported the same effects for behavioral and neural measures. A likely reason is that researchers have tested these measures at different narrow ranges of SNRs as well as in different participants which makes a direct comparison between neural and behavioral measures difficult. In the present study, we minimized these experimental biases by evaluating our behavioral and neural measures under the same SNR conditions and in the same participants. We hypothesize that if the single-task, dual-task, phase synchronization 
and alpha power measure are sensitive to changes in processing load, our results will show a peak-shaped behavior across a wide range of SNRs. For the self-report measure, we expect that perceived effort is highest at the low SNRs and decreases with increasing SNR.

The second aim of this study involves how much each listening effort measure accounts for the unexplained variability in envelope tracking beyond speech understanding. Previous studies have shown that envelope tracking has the potential to be used as an objective measure of speech understanding (Vanthornhout et al., 2018; Decruy et al., 2019; Lesenfants et al., 2019; Verschueren et al., 2019). However, young NH participants show differences in neural envelope tracking that can not be fully attributed to changes in SNR or speech understanding. In addition, recent studies have observed a lower envelope tracking for equally intelligible sentences presented without a masker compared to sentences presented at a high SNR (Das et al., 2018; Lesenfants et al., 2019). We hypothesize that these two unexplained differences in envelope tracking are related to changes in listening effort based on the findings of following behavioral studies. Wu et al. (2016) have found that listening effort, measured using a dual-task, is generally characterized by a peak-shaped behavior. When assessing the individual results, however, they found that the shape of the curve varied substantially across participants. In addition, Houben et al. (2013) have shown that participants achieve similar speech understanding for sentences presented without a masker compared to sentences presented at $+4 \mathrm{~dB}$ SNR, but expended significantly less effort for the condition without a masker. Besides this, it is suggested that the delta and theta frequency band play different roles in the encoding of speech (Ding \& Simon, 2014; Etard \& Reichenbach, 2019; Lesenfants et al., 2019). Since the precise role remains debated, we also investigated if listening effort modulates neural envelope tracking for both frequency bands or only for the delta- or theta-band.

\section{Material and methods}

\section{Participants}

Thirteen Flemish speaking participants, aged between 17 and 28 years, participated in this study. All participants had normal hearing as their pure tone thresholds were better than 25 dB HL at all octave frequencies in both ears $(125 \mathrm{~Hz}$ up to $8 \mathrm{kHz})$. During a short interview, the participant's medical history and education were questioned because it is known that serious concussions, medication for the treatment of insomnia (Van Lier et al., 2004) and learning disabilities such as dyslexia (Power et al., 2016; De Vos et al., 2017) can affect brain responses. The hand and ear preference of the participants were also determined using a 
Flemish, modified version of the laterality preference inventory of Coren (1993). Lastly, a word reading test, cognitive screening test and working memory test were administered to assess reading and cognitive skills which are needed for completing the secondary task (see Dual-task paradigm). With regard to the word reading test (één-minuut test; Brus \& Voeten, 1973), all participants obtained a score higher than percentile 10, indicating no persistent reading and/or spelling difficulties. With regard to the cognitive tests, all participants passed the cognitive screening test $(>25 / 30$ on Montreal Cognitive Assessment; Nasreddine et al., 2005) and achieved a score higher than 27/60 on the working memory test (Flemish computerized version of Reading Span Test, RST; Van Den Noort et al., 2008; Vercammen et al., 2017). This study was approved by the Medical Ethics Committee UZ KU Leuven / Research (reference no. S57102 (Belg. Regnr: B322201422186) and S58970 (Belg. Regnr: B322201629016)). All participants took part voluntarily and gave their written informed consent.

\section{Behavioral experiment}

After screening, participants first completed the behavioral experiment that took approximately two hours (overview figure 1). For 12 of the 13 participants, this took place at the research group ExpORL of the KU Leuven, in a double-walled, soundproof booth. For one participant, the behavioral experiment took place in a quiet room at their home because time and distance restrictions did not allow the participant to come to the lab twice. First, the subject-specific SNRs were determined. Second, the speech-in-noise test and verbal working memory test were administered simultaneously in a dual-task paradigm. In the following sections, the tests and two behavioral measures of listening effort are described in more detail. 
A

Behavioral experiment

Determination of subject-specific SNRs

* Measure percentage correctly identified sentences at three levels (dots) and fit psychometric curve

* Infer 6 subject-specific SNRs (triangles: 20\%, 35\%, 50\%, $65 \%, 80 \%$ and $95 \%$ )

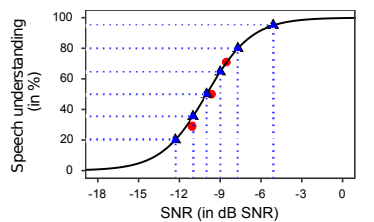

Recall matrix sentences during dual-task paradigm $\times 7$ speech understanding levels

* Reaction time to matrix sentences (RTprim)

* Reaction time to memorized words (RTsecond)

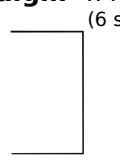
6 subject-specific SNRs and No noise

* Percentage correctly identified sentences

\section{Behavioral measures} of listening effort

EEG experiment

$\begin{array}{ll}\text { Listen to concatenated sentences } & \times 7 \text { speech understanding levels } \\ & \text { (6 subject-specific SNRs and No noise) }\end{array}$

* Neural envelope tracking

* Rate effort expended during listening (0-100\%)

* Phase synchronization

* Alpha power

Self-report measure of listening effort

Neural measures of listening effort

* Rate speech understanding of sentences (0-100\%)

Figure 1: Overview of study design.

\section{Determination of a wide range of subject-specific SNRs}

In this study, we wanted to investigate listening effort across a wide range of SNRs because recent studies demonstrated a peak-shaped behavior of listening effort as a function of SNR (Zekveld \& Kramer, 2014; Wu et al., 2016; Ohlenforst, Zekveld, \& Lunner et al., 2017). To ensure this, six subject-specific SNRs were determined prior to the dual-task paradigm. The Flemish matrix sentence test (Luts et al., 2014) was used as speech material and consists of sentences with a fixed syntactical structure of 5 word categories "name, verb, numeral, color and object" (e.g. "Emma buys three blue bikes"). Participants were instructed to recall the sentences out loud using a 5 x 10 matrix containing 10 possibilities for each word of the sentence. We motivated participants to guess based on the matrix when it was more difficult to understand the sentences. When participants did not know the answer, they could also choose to give no answer. The percentage of correctly repeated words per list/SNR was calculated to obtain a speech understanding score.

To obtain the SNRs, the matrix sentences were masked with a stationary speech-weighted noise with the same long-term average spectrum as the matrix sentences. Three adaptive procedures (Brand \& Kollmeier, 2002) converging to 29\%, 50\% and 71\% speech understanding 
(SU) were administered to determine the six subject-specific SNRs. This was done by using the result of each adaptive procedure to fit a sigmoid function per participant using the following formula $S(S N R)=\gamma+(1-\gamma-\lambda) \frac{1}{1+e^{-\frac{(S N R-\alpha)}{\beta}}}$ with $\gamma$ the guess-rate fixed to zero, $\lambda$ the lapse-rate fixed to zero, $\alpha$ the midpoint and $\beta$ the slope. From this fit, we consequently inferred a subject-specific SNR per SU-level (20\%, 35\%, 50\%, 65\%, 80\%, 95\% SU). These subject-specific SNRs were used for both the behavioral and EEG experiment.

\section{Dual-task paradigm}

We created a new dual-task paradigm in which both tasks require the allocation of similar cognitive resources: 1) the primary task is a standardized speech-in-noise test and 2) the secondary task is a verbal working memory test, especially developed for this study.

During the dual-task paradigm, participants were instructed to prioritize the primary speechin-noise task to ensure that the performance on the secondary task reflects the effort expended on the primary task (limited cognitive capacity theory of Kahneman, 1973). As illustrated in figure 2, a dual-task trial consisted of three screens. First, participants were instructed to read and remember three consonant-vowel-consonant (CVC) words (e.g. "cat, hut, pen") which were presented on a computer screen for three seconds. Second, a blank screen appeared, a matrix sentence was played and participants were asked to recall the sentence out loud. Third, one of the three CVC words reappeared, e.g. "pen" and participants were instructed to push, as quickly and accurately as possible, the button that corresponded with the original position at which the word was presented, e.g. "pen: third position". Per trial, it was randomly chosen which word reappeared. Note that this secondary task also relies on short-term and visuospatial working memory since participants are instructed to remember the words and their position on the screen. Nevertheless, the explicit instruction to read the words, activates verbal working memory which is the same cognitive function that speech-in-noise tests rely on. In view of this, our test is likely to be more sensitive to changes in listening effort than other visual tasks used in dual-task paradigms (Picou \& Ricketts, 2014; Gagné et al., 2017). Since studies have shown that reaction times during single speech-in-noise tasks can be used as a behavioral correlate of listening effort (Gatehouse \& Gordon, 1990; Pals et al., 2015), we asked the participants to push the middle button when recalling the matrix sentence (second screen). The reaction time (RTprim) was calculated between the end of the auditory presentation of the sentence and the recall of the participant (i.e. when the participant pushes the middle button). In dual-task studies, often the reaction time on the secondary task is used as a measure of listening effort (Anderson Gosselin \& Gagné, 2010; Gagné et al., 2017). In this study, the reaction time (RTsecond) was calculated as the time between the 
reappearance of the $\mathrm{CVC}$ word and the answer of the participant (i.e. when the participant pushes the button corresponding with the original position). Similar to Houben et al. (2013), we included both correct and incorrect trials because we did not want to remove valuable information about the difficult secondary task conditions. To assess listening effort as a function of speech understanding, six matrix sentence lists of each 20 dual-task trials were administered at six fixed, subject-specific SNRs (20\%, 35\%, 50\%, 65\%, 80\%, 95\% SU) and one list was presented without a masker (No noise).

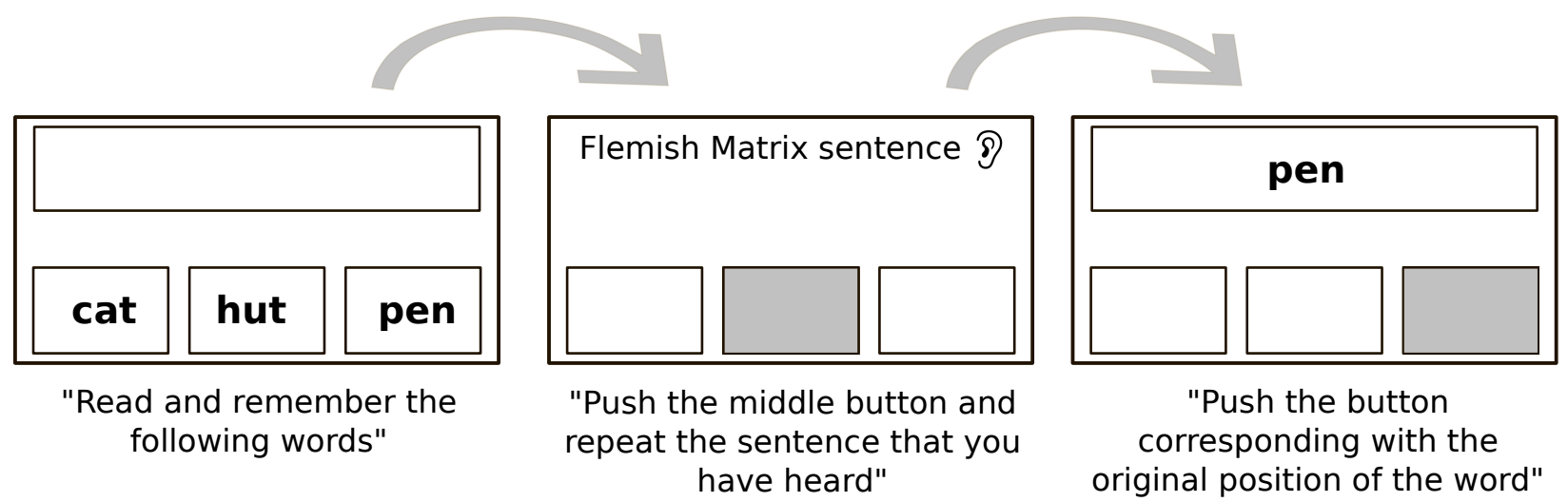

Figure 2: Overview of the dual-task paradigm. Per trial three screens were presented. First, three CVC words appear, then a matrix sentence is presented and finally, one of the three words reappears.

Prior to the dual-task paradigm, the speech-in-noise test and verbal working memory test were first administered separately in a single-task paradigm to exclude the bias due to procedural learning for the dual-task paradigm. Similarly to the dual-task paradigm, seven lists of the matrix sentence test were administered (six subject-specific SNRs and No noise). Participants also completed 10 runs of 10 trials of the verbal working memory test, because pilot results showed no further improvement in reaction times after 100 trials.

\section{Reaction time preprocessing}

Using reaction times as an outcome measure often requires several pre-processing steps because reaction times are not normally distributed but are always positive and skewed to the right. In this study, we pre-processed our reaction times in three steps. First, reaction times shorter than $200 \mathrm{~ms}$ (Whelan, 2008) were removed as well as reaction times longer than $2.24 *(M A D / 0.6745)+M$ with $M A D$ defined as the median absolute deviation and $M$ being the sample median (MAD-median rule: Wilcox et al., 2013). Second, we calculated 
the median of the remaining reaction times across trials, per SU-level and per participant (Ratcliff, 1993). Finally, we normalized the reaction times per participant in a last step by subtracting the median for the "No noise" condition from each median value of the SU-level condition and dividing this by the median of the "No noise" condition (Houben et al., 2013;

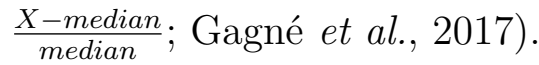

\section{Auditory and visual stimulation}

For the presentation of the matrix sentences, we used a laptop connected to a RME Hammerfall DSP Multiface II or RME Fireface UC soundcard (RME, Haimhausen, Germany). The sentences were presented using the software platform APEX (Dept. Neurosciences, KU Leuven) (Francart et al., 2008). For 8 of the 13 participants, the target speech stimuli and masker were presented diotically through ER-3A insert phones (Etymotic Research, Inc., IL, USA). For the remaining 5 participants, the stimuli were presented monaurally to the right ear. To create the subject-specific SNRs, the level of the noise was adjusted while the speech level was fixed at an intensity of 62 or $65 \mathrm{~dB}$ SPL (A weighted) for diotic and monaural stimulation respectively. Before administering the experiments, all stimuli were first calibrated with a type 2260 sound level pressure meter, a type 4189 half-inch microphone and a 2cc coupler (Bruel \& Kjaer, Copenhagen, Denmark). Similar to matrix sentences for the primary task, the three CVC-words for the secondary task were presented using the software platform APEX. The reaction times during the verbal working memory task were registered using APEX in combination with three large buttons mounted on a wooden board. At an equal distance of each button, a hand was drawn on the wooden board to indicate the place at which participants could place their preference hand for pushing the buttons.

\section{EEG experiment}

After the behavioral experiment, an EEG experiment of approximately two and a half hours was conducted to investigate the association between neural envelope tracking and listening effort (overview figure 1). During the EEG, participants listened to matrix sentences, presented at the subject-specific SNRs as during the behavioral experiment. This way, we could link the two behavioral listening effort measures (RTprim and RTsecond; see Behavioral

experiment) with envelope tracking. Since listening effort can also be measured using selfreport or EEG (Pichora-Fuller et al., 2016), we collected three additional listening effort measures (self-report, phase synchronization and alpha power) during the EEG experiment. In the next section, the procedure of the EEG experiment, the self-report and neural effort 
measures as well as the signal processing using MATLAB R2016b (Mathworks, Natwick, USA) are described in more detail.

\section{Neural envelope tracking and self-reported listening effort}

To measure envelope tracking, we presented a concatenation of 40 matrix sentences at the same seven SNRs as the dual-task (six subject-specific SNRs and No noise). Between the sentences, a silent gap that was uniformly distributed between 0.8 and $1.2 \mathrm{~s}$, was inserted. The participants listened to three repetitions of the same matrix sentences at the seven SNRs (3 repetitions $\mathrm{x} 7 \mathrm{SNRs}=$ total of 21 conditions). Since matrix sentences do not contain context and are difficult to remember, repeated listening should not have introduced a bias of content learning on our results. The order of the SNRs was quasi-randomized across repetitions and participants. To keep them attentive, a question was asked per condition (e.g. "How many red boats did Jacob carry?"). After the question, participants were instructed to rate 1) their perceived understanding and 2) the effort they had to expend during listening by reporting a percentage between 0 and $100 \%$. To better compare the five effort measures, the effort ratings were normalized per repetition similarly to the reaction times. More specifically, per participant and per SU-level, the value for the "No noise" condition was subtracted from each rating. We did not divide by the value of the "No noise" condition because many participants rated the "No noise" condition equal to zero, i.e. low effort. Lastly, the story "Milan" by Stijn Vranken (8 subjects) or the story "De Wilde Zwanen" by Hans Christian Andersen (5 subjects) was presented without a masker to train the linear decoder (see Signal Processing: Envelope reconstruction).

\section{Signal processing: Envelope reconstruction}

Neural envelope tracking was measured by correlating the original envelope of the matrix sentences with the envelope reconstructed from the EEG responses. To obtain this, the original speech envelope was first extracted by filtering the target speech stimulus using a Gammatone filterbank followed by a power law (Biesmans et al., 2017). Next, the envelope was downsampled from $44100 \mathrm{~Hz}$ to $256 \mathrm{~Hz}$ to decrease processing time. Then, a type 2 zero-phase Chebyshev filter (with $80 \mathrm{~dB}$ attenuation at $10 \%$ outside the passband) was applied to the envelope. For the delta-band, we filtered from 1 up to $4 \mathrm{~Hz}$, for the theta-band from 4 up to $8 \mathrm{~Hz}$. Finally, the speech envelope was further downsampled to $128 \mathrm{~Hz}$.

The EEG data was downsampled from $8192 \mathrm{~Hz}$ to $256 \mathrm{~Hz}$ similarly to the envelope. Next, a generic EEG artifact removal algorithm (Somers et al., 2018) was applied on the EEG-data to remove eye artifacts similar to described in Decruy et al. (2019). EEG-signals were then 
re-referenced to $\mathrm{Cz}$ and bandpass filtered for delta- and theta-band using the same Chebyshev filter as used for the envelope. In a last step, the EEG was downsampled to $128 \mathrm{~Hz}$.

To reconstruct the envelope from the EEG, we used the stimulus reconstruction approach described by Vanthornhout et al. (2018). More specifically, we used a linear decoder or spatiotemporal filter that linearly combines the EEG signals of the different channels and their time shifted versions to optimally reconstruct the envelope. Mathematically, this can be formulated as follows: $\hat{s}(t)=\sum_{n} \sum_{\tau} g(n, \tau) R(t+\tau, n)$, with $\hat{s}(t)$ the reconstructed envelope, $t$ the time ranging from 0 to $\mathrm{T}, n$ the index of the recording electrode ranging from 1 to 63 , $\tau$ the post-stimulus integration window length, $g$ the linear decoder and $R$ the shifted neural responses. We chose an integration window from 0 until $250 \mathrm{~ms}$. The weights of the decoder were determined in a training phase by applying ridge regression with regularization on the inverse autocorrelation matrix: $g=\left(R R^{T}\right)^{-1}\left(R S^{T}\right)$ with $R$ as the time-lagged matrix of the EEG signal and $S$ the speech envelope (inspired on the mTRF toolbox: Lalor et al., 2006, 2009). The decoder $g$ was trained for each participant on the story "De Wilde Zwanen" or "Milan".

After training, the subject-specific decoder was applied on the EEG responses to the matrix sentences, to reconstruct the envelope for each presentation and SNR. This reconstructed envelope was then correlated with the actual envelope using a bootstrapped Spearman correlation after removing the parts that contained silences. Neural envelope tracking per participant and per SNR condition was measured by calculating the mean of the correlations of the three presentations. Furthermore, we calculated a significance level for envelope tracking in order to interpret the individual data points of a particular participant. We did not use this significance level to exclude data points from statistical analysis. The significance level was calculated by correlating random permutations of the actual and reconstructed envelope (1000 times) and taking percentile 2.5 and 97.5 to obtain a $95 \%$ confidence interval.

\section{Phase synchronization and alpha power}

Next to RTprim, RTsecond and self-reported effort, we included two additional neural measures of listening effort in the present study. The phase synchronization of EEG responses was calculated based on the method of Bernarding et al. (2014). They proposed that when a person listens to speech, the distribution of the instantaneous phase of ongoing neural activity reflects listening effort. Alpha power was extracted using Thomson's multitapers spectral analysis (Thomson, 1982). In the following section, the several signal processing steps are described in more detail. 


\section{Signal Processing: Phase synchronization and alpha power}

The EEG was first downsampled from $8192 \mathrm{~Hz}$ to $512 \mathrm{~Hz}$, similar to Bernarding et al. (2014). Then artifacts were removed using a generic EEG artifact removal algorithm (Somers et al., 2018). Consequently, EEG signals were re-referenced to $\mathrm{Cz}$ and bandpass filtered using the same Chebyshev filter as used for envelope reconstruction. In contrast to neural envelope tracking, we did not limit the filtering to the delta- or theta-band but selected a wide range of frequencies, from 1 up to $40 \mathrm{~Hz}$, similar to Bernarding et al. (2014).

Phase synchronization of the ongoing EEG activity was evaluated across time by computing the distribution of the instantaneous phases of ongoing neural EEG activity on the unit circle, per SU-level (Bernarding et al., 2014). First, we extracted the instantaneous phase per time sample (approximately $120 \mathrm{~s}$ of EEG-data per SNR x $512 \mathrm{~Hz}=61440$ samples) using a continuous wavelet transform (scale $\mathrm{a}=40$ represents $7.68 \mathrm{~Hz}$ time interval of $\mathrm{N} 1$ component; Morlet wavelet). Since, we were only interested in the phase synchronization to the stimulus, we removed the instantaneous phases for the silent gaps that were inserted between the matrix sentences. Second, we evaluated the distribution of the remaining instantaneous phases for electrode TP8 (i.e., closest to the right mastoid) on the unit circle using a Rayleigh Test (Strauss et al., 2010; Bernarding et al., 2014). Therefore, we first computed the mean resultant vector of the phase values for $\mathrm{n}$ time samples: $\bar{R}=\sqrt{\bar{C}^{2}+\bar{S}^{2}}$ in which $\bar{C}=\frac{1}{n} \sum_{i=1}^{n} \cos \varphi_{i}$ and $\bar{S}=\frac{1}{n} \sum_{i=1}^{n} \sin \varphi_{i}$. Then, we approximated the probability $\operatorname{Pr}$ that the phases are uniformly distributed using $\tilde{P r}=e^{\sqrt{1+4 n+4\left(n^{2}-(n \bar{R})^{2}\right)}-(1+2 n)}$. If the phases are dispersed around the unit circle, the mean resultant vector $\bar{R}$ is small, resulting in a small probability to reject the null hypothesis of the test that the data are uniformly distributed. Finally, we calculated phase synchronization as $1-\tilde{P r}$. During the statistical analyses, we observed a large variability of the phase synchronization values across participants. Because this could be due to attentional effects in the beginning and end of each condition, we divided each condition in three equal time sequences and only included the phase values of the middle part to calculate the mean resultant vector. Because we are interested in the changes in effort between different SU-levels, i.e. changes in phase synchronization as a function of speech understanding instead of time, excluding the beginning and end parts of each condition does not bias our results of interest.

Alpha power was calculated by extracting the power density of the EEG for frequencies between 8 and $15 \mathrm{~Hz}$. We chose a broad frequency range because alpha power can peak at a different frequencies between participants. Per SNR condition or SU-level, power density for this frequency range was extracted from the EEG measured during presentation of matrix sentences. This was done over consecutive non-overlapping 1-s epochs, using Thomson Multitapers Spectral Analysis (7 tapers; Thomson, 1982). Topoplots on which the median of alpha power across epochs was plotted, showed only changes across SU-levels for the 
parietal-occipital electrodes. Because of this, we selected a subset of electrodes (P9, P7, P5, PO3, POz, PO4, P6, P8, P10, PO7, O1, O2, Oz, PO8, Iz) and used the median alpha power across this selection and time course as our second neural effort measure.

Finally, phase synchronization as well as alpha power were normalized per repetition, SNR and participant. In previous studies, alpha power was often normalized against a baseline period before the stimulation (Obleser et al., 2012; Dimitrijevic et al., 2019). We did not do this because we wanted to compare the five effort measures, so we normalized each measure the same way, i.e. each median value of a SU-level condition was subtracted and divided by the median of the "No noise" condition.

\section{EEG recording}

The EEG experiment was administered for all participants at the research group in a triplewalled, soundproof booth, equipped with a Faraday cage to avoid electromagnetic interference. The same auditory stimulation set-up as the behavioral experiment, was used to present the matrix sentences to the participants. More specifically, stimuli were presented to 8 of the 13 participants diotically through ER-3A insert phones and for the remaining 5 participants, sentences and masker were presented monaurally, to the right ear. To measure the EEG, we mounted 64 active $\mathrm{Ag} / \mathrm{AgCl}$ electrodes and two extra electrodes, serving as the common electrode (CMS) and current return path (DRL), in head caps according to the 10-20 electrode system. The EEG recordings were conducted using the BioSemi ActiveTwo system (Amsterdam, Netherlands), digitized at a sampling rate of $8192 \mathrm{~Hz}$ and stored on a hard disk using the BioSemi ActiView software.

\section{Statistical analyses}

The statistical analyses were conducted using $\mathrm{R}$ software (version 3.6.3; nlme package version 3.1.144; Field et al., 2012; Pinheiro et al., 2017). We investigated the association between the different listening effort measures and their link with envelope tracking using Linear Mixed-effect Models (LMMs). The fixed-effect part of the LMMs consisted of the predictors of interest whereas the random-effect part included the variable participant, nested in the repeated measure predictor SU-level if this improved the model fit. For the comparison of the different effort measures as a function of speech understanding, we used polynomial (linear, quadratic and cubic) terms in our LMMs as previous studies have shown a non-linear relationship, i.e. peak-shaped behavior, between effort and SNR (Wu et al., 2016; Ohlenforst, Zekveld, \& Lunner et al., 2017). All models were fitted using the maximum likelihood 
estimation. The best fitting model was determined by first progressively introducing multiple fixed-effects and corresponding interactions. Then, the different models were compared using likelihood ratio tests and Akaike's Information Criterion (AIC; Akaike, 1974). The best fitting model served as starting point for the evaluation of the contribution of other predictors until the final best fitted model was determined. Significant main and interaction effects of the final model are discussed in the results section by reporting the unstandardized regression coefficient $(\beta)$ with standard error (SE), degrees of freedom (df), t-Ratio and $\mathrm{p}$-value per fixed-effect term. A significance level of $\alpha=0.05$ was set for all the models.

In addition to p-values, we calculated effect sizes and Bayes factors. The standardized effect size for the fixed-effect terms of our LMMs were determined using Cohen's $f^{2}$ (Selya et al., 2012). To interpret the effect size, we followed the guidelines of Cohen (1988): values of Cohen's $f^{2} \geq 0.02, f^{2} \geq 0.15$, and $f^{2} \geq 0.35$ represent small, medium, and large effect sizes, respectively. Cohen's $f^{2}$ was calculated as follows: $\frac{R_{A B}^{2}-R_{A}^{2}}{1-R_{A B}^{2}}$ where $R_{A B}^{2}$ is the variance explained by the variable $\mathrm{A}$ and $\mathrm{B}$ relative to a model without regressors and $R_{A}^{2}$ is the variance explained by the variable A relative to a model without regressors (Selya et al., 2012). When the model did not include multiple variables, Cohen's $f^{2}$ was calculated as follows: $\frac{R_{A}^{2}}{1-R_{A}^{2}}$ with $R_{A}^{2}$ the variance of the variable A relative to the null model without regressors. We used the Multi-Model Inference $\mathrm{R}$ package (Nakagawa et al., 2017; version 1.43.15; Barton, 2019) to calculate the conditional Pseudo- $\mathrm{R}^{2}$, i.e. variance explained by the fixed and random effects of a LMM. In addition to effect sizes, we also calculated Bayes factors. We define the Bayes factor as the ratio of the probability of the data given the alternative $\left(H_{1}\right)$ versus null $\left(H_{0}\right)$ hypothesis or $B F_{10}=\frac{f\left(\text { data } \mid H_{1}\right)}{f\left(\text { data } \mid H_{0}\right)}$ (Rouder et al., 2009; Etz et al., 2018). For most of our results, the alternative hypothesis model is an LMM that contains the fixed-effect of interest and the null hypothesis model is an LMM without regressors. If the null hypothesis model contains fixed-effect terms, this will be specified. We followed the guidelines of Jeffreys (Jeffreys, 1961; cited in Stefan et al., 2019) to interpret the Bayes factor: $B F_{10}>100$ (extreme evidence for $H_{1}$ ), 30-100 (very strong evidence for $H_{1}$ ), 10-30 (strong evidence for $H_{1}$ ), 3-10 (moderate evidence for $H_{1}$ ), 1-3 (anecdotal evidence for $H_{1}$ ), 1 (no evidence). The inverse of these values yield the same interpretation for $H_{0}$. We used the BayesFactor R package which employs the JZS approach to set the priors and calculate the Bayes factors for LMMs (Jarosz \& Wiley, 2014; version 0.9.12.4.2; Morey, 2019). 


\section{Results}

\section{Listening effort as a function of speech understanding}

Prior to linking each effort measure with neural envelope tracking, we first compared the different listening effort measures based on how they behave as a function of speech understanding.

\section{Speech-in-noise performance}

The five listening effort measures used in this study were collected during different experiments. RTprim and RTsecond were collected during the behavioral experiment whereas self-report, phase synchronization and alpha power were registered during the EEG experiment. Although we used the same subject-specific SNRs for each experiment, differences in speech understanding performance could result in differences between the measures. We investigated this by comparing the speech understanding scores obtained during three procedures: the recall scores during the speech-in-noise test administered as a (1) single-task paradigm (2) or dual-task paradigm as well as (3) the rated scores obtained during the EEG experiment. The psychometric functions, plotted in figure 3, show that similar speech understanding scores were obtained for the three procedures. Accordingly, no significant main effect was detected by an LMM with procedure as fixed-effect term and participant nested in SU-level as random-effect term $(\mathrm{p}>0.05)$. In addition, the estimated Bayes factor indicated strong evidence for $H_{0}$, i.e. similar speech understanding performance among the three procedures $\left(B F_{10}=0.04\right)$. Similarly, Holm adjusted posthoc tests did not yield significant differences between the different procedures (single-task versus dual-task: $\mathrm{p}=0.173$; single-task versus EEG: $\mathrm{p}=0.584$ ); dual-task versus EEG: $\mathrm{p}=0.584$ ). Therefore, we will not make a distinction between the recall versus rated speech understanding scores for the following analyses. 


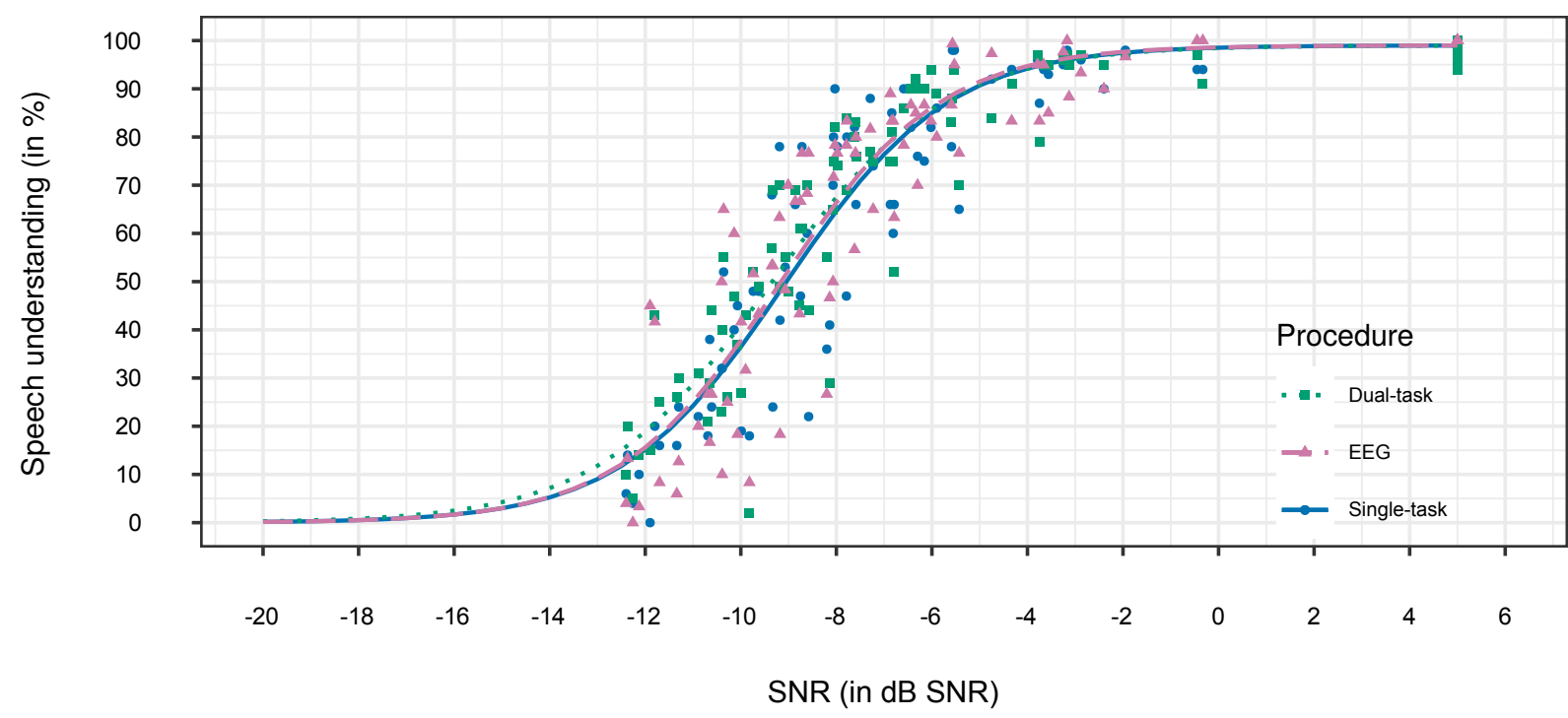

Figure 3: Comparison of speech in noise performance of 13 participants measured during the single-task paradigm (recall), dual-task paradigm (recall) and EEG experiment (rate). For each procedure, a psychometric function (color-coded) is fitted on the data of all participants across the six subject-specific SNRs and No noise condition (for visualization No noise condition is plotted at $5 \mathrm{~dB}$ SNR instead of $100 \mathrm{~dB}$ SNR). No significant difference was found between the different procedures.

\section{Behavioral, self-report and neural measures of listening effort}

To compare the five listening effort measures, we analyzed each outcome measure as a function of the speech understanding scores obtained at the seven SNRs (six subject-specific SNRs and No noise). Per measure, we built an LMM with speech understanding as fixed-effect term (table 1; figure 4). For the behavioral experiment, we detected a supralinear decrease in RTprim with increasing speech understanding $(\beta=-2.51 \mathrm{e}-04, \mathrm{SE}=3.88 \mathrm{e}-05, \mathrm{p}<0.001$, Cohen's $f^{2}$ (large effect) $\left.=0.3636\right)$. Moreover, RTprim was longer at unfavorable SU-levels (0-30\%) and then became shorter, and thus better, with increasing speech understanding (see figure 4, left panel). RTsecond, on the other hand, did not decrease as a function of speech understanding, but had a peak-shape (revealed by a significant cubic and linear fixed-effect term; cubic: $\beta=-9.22 \mathrm{e}-07, \mathrm{SE}=2.53 \mathrm{e}-07, \mathrm{p}<0.001$; linear: $\beta=7.31 \mathrm{e}-03$, SE $=3.23 \mathrm{e}-03, \mathrm{p}=0.026$, Cohen's $f^{2}$ (medium effect) $\left.=0.3382\right)$. This can also be inferred from figure 4 in which RTsecond first increased from unfavorable SU-levels (0-10\%; linear term) until intermediate SU-levels (40-60\%; cubic term), and then decreased with increasing 
speech understanding (80-100\%; cubic term). Further support for these effects can be derived from the estimated Bayes factors revealing extreme evidence for $H_{1}\left(B F_{10}(\right.$ RTprim $)>1000$; $B F_{10}(R T$ second $\left.)>1000\right)$. Taken together, RTprim and RTsecond showed a different effect of listening effort as function of speech understanding.

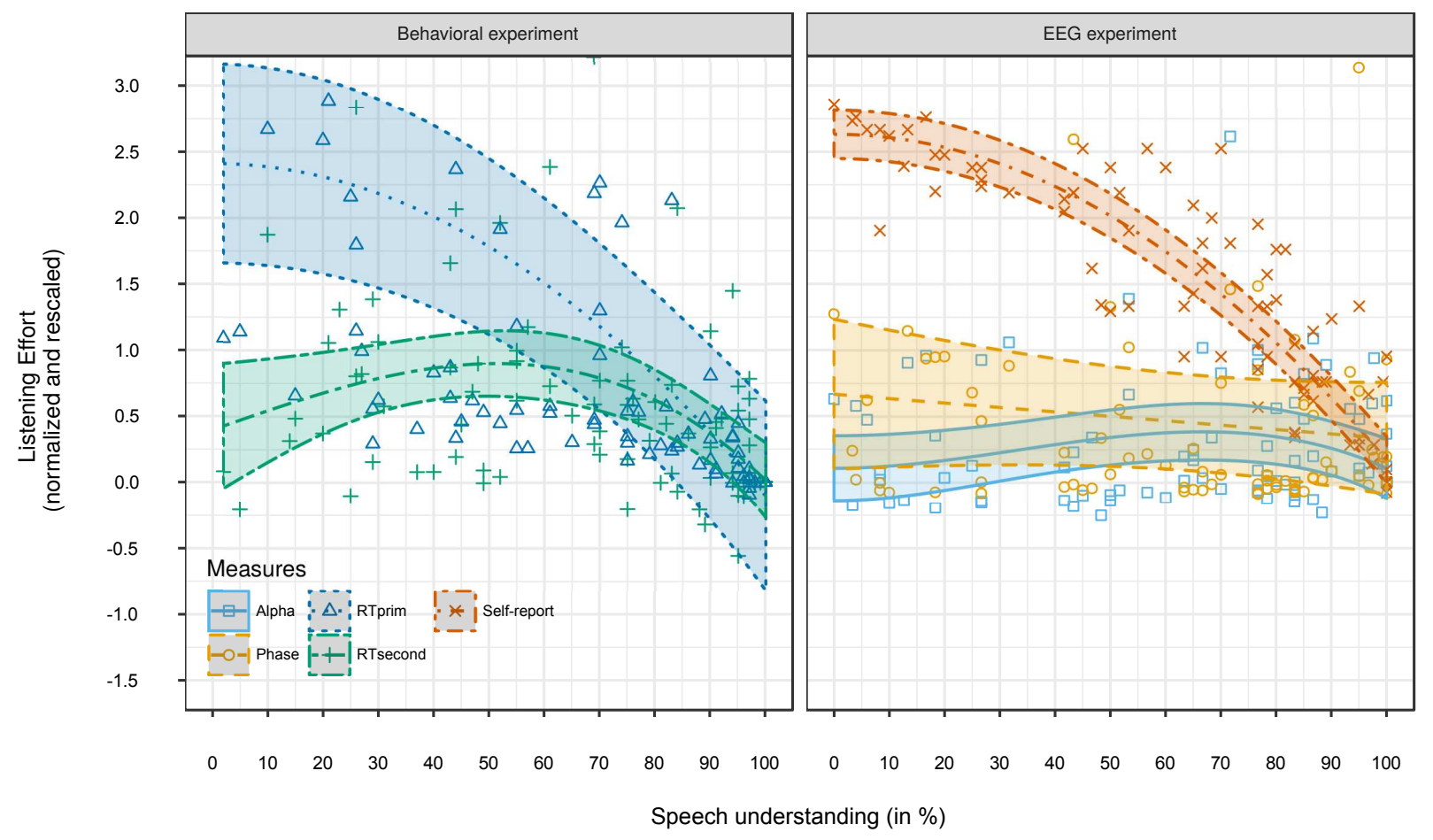

Figure 4: Listening effort as a function of speech understanding for two measures obtained during the behavioral experiment (RTprim and RTsecond, left panel) and for three measures obtained during the EEG experiment (self-report, phase synchronization and alpha power, right panel). For each measure, a regression line with confidence intervals (shaded areas; color-coded) was fitted on the data. The regression lines indicate that different measures reflect different aspects of listening effort (see table 1).

From the EEG experiment, we collected three listening effort measures. Similar to RTprim, self-reported effort decreased supralinearly with increasing speech understanding $(\beta=$ 8.62e-03, SE $=2.83 \mathrm{e}-04, \mathrm{p}<0.001$, Cohen's $f^{2}$ (large effect) $=12.0027$, see also right panel of figure 4). The estimated Bayes factor for self-reported effort revealed extreme evidence for $H_{1}$, indicating that self-reported effort is modulated by speech understanding $\left(B F_{10}(\right.$ Self - report $\left.)>1000\right)$. The neural measures of listening effort behaved differently. More specifically, the LMM for phase synchronization detected no significant effect of speech 
understanding $\left(\mathrm{p}=0.318\right.$, Cohen's $f^{2}$ (no effect) $\left.=0.0015\right)$. The estimated Bayes factor for phase synchronization revealed only anecdotal evidence for $H_{0}$, suggesting that the data is uninformative $\left(B F_{10}(\right.$ Phase $\left.)=0.34\right)$. Lastly, the LMM for alpha detected a peak-shape as a function of speech understanding (revealed by a significant cubic and quadratic fixed-effect term; cubic: $\beta=-9.32 \mathrm{e}-07, \mathrm{SE}=3.06 \mathrm{e}-07, \mathrm{p}=0.003$; quadratic: $\beta=9.31 \mathrm{e}-05, \mathrm{SE}=3.20 \mathrm{e}-05$, $\mathrm{p}=0.005$, Cohen's $f^{2}$ (small effect) $=0.0966$; figure 4 ). More specifically, alpha power was highest between $60 \%$ and $80 \%$ speech understanding. The estimated Bayes factor revealed moderate evidence for $H_{1}$, indicating that it is likely that speech understanding affects alpha power $\left(B F_{10}(\right.$ Alpha $\left.)=3.77\right)$.

Table 1: Linear Mixed-effect Models: The effect of speech understanding on five listening effort measures (RTprim, RTsecond, Self-report, Phase synchronization and Alpha power). Regression coefficients ( $\beta$ values), standard errors (SE), degrees of freedom (df), t-Ratios and p-values are reported per fixed-effect term. Participant was included as a random effect.

\begin{tabular}{|c|c|c|c|c|c|c|}
\hline Behavioral & experiment & & & & & \\
\hline Measure & Fixed-effect terms & $\beta$ value & $\mathrm{SE}$ & $\mathrm{df}$ & t-Ratio & $\mathrm{p}$-value \\
\hline \multirow[t]{2}{*}{ RTprim } & Intercept & 2.41 & 0.38 & 77 & 6.33 & $<0.001$ \\
\hline & $\mathrm{SU}^{2}$ & $-2.51 \mathrm{e}-04$ & $3.88 \mathrm{e}-05$ & 77 & -6.47 & $<0.001$ \\
\hline \multirow[t]{3}{*}{ RTsecond } & Intercept & 0.20 & 0.13 & 76 & 1.58 & 0.117 \\
\hline & SU & $7.31 \mathrm{e}-03$ & $3.23 \mathrm{e}-03$ & 76 & 2.27 & 0.026 \\
\hline & $\mathrm{SU}^{3}$ & $-9.22 \mathrm{e}-07$ & $2.53 \mathrm{e}-07$ & 76 & -3.65 & $<0.001$ \\
\hline EEG & experiment & & & & & \\
\hline Measure & Fixed-effect terms & $\beta$ value & SE & $\mathrm{df}$ & t-Ratio & $\mathrm{p}$-value \\
\hline \multirow[t]{2}{*}{ Self-report } & Intercept & 92.11 & 3.25 & 77 & 28.34 & $<0.001$ \\
\hline & $\mathrm{SU}^{2}$ & $-8.62 \mathrm{e}-03$ & $2.83 \mathrm{e}-04$ & 77 & -30.41 & $<0.001$ \\
\hline \multirow[t]{2}{*}{ Phase } & Intercept & 6.69 & 2.87 & 77 & 2.33 & 0.023 \\
\hline & $\mathrm{SU}$ & -0.03 & 0.03 & 77 & -1.00 & 0.318 \\
\hline \multirow[t]{3}{*}{ Alpha } & Intercept & 0.05 & 0.06 & 76 & 0.87 & 0.385 \\
\hline & $\mathrm{SU}^{2}$ & $9.31 \mathrm{e}-05$ & $3.20 \mathrm{e}-05$ & 76 & 2.91 & 0.005 \\
\hline & $\mathrm{SU}^{3}$ & $-9.32 \mathrm{e}-07$ & $3.06 \mathrm{e}-07$ & 76 & -3.04 & 0.003 \\
\hline
\end{tabular}

To relate the normalized values of the different effort measures to the original units, the median original values across participants, for each SU-level and effort measure, are shown in table 2. Similar to our statistical analyses on the normalized values, it can be inferred that RTsecond and alpha power have highest values around intermediate speech understanding 
levels (peak-shape) whereas RTprim and self-reported effort ratings are highest for the most difficult condition (20\% SU) and then decrease with increasing speech understanding.

Table 2: Per speech understanding level (SU-level), the median original values across participants for each effort measure are reported (reaction time on primary task (RTprim), reaction time on secondary task (RTsecond), self-reported effort (Self-report), phase synchronization (Phase) and alpha power (Alpha)).

\begin{tabular}{llllll}
\hline SU-level & RTprim $(\mathrm{ms})$ & RTsecond $(\mathrm{ms})$ & Self-report $(\%)$ & Phase $(\%)$ & Alpha $\left(\mu V^{2}\right.$ per Hz) \\
\hline $20 \%$ & 2596.00 & 1076.00 & 90.00 & 21.40 & 0.99 \\
$35 \%$ & 2077.00 & 1255.00 & 80.00 & 24.05 & 0.99 \\
$50 \%$ & 2371.00 & 1504.00 & 70.00 & 15.74 & 1.11 \\
$65 \%$ & 2065.00 & 1081.00 & 50.00 & 15.21 & 1.05 \\
$80 \%$ & 1821.50 & 1034.00 & 30.00 & 10.37 & 1.07 \\
$95 \%$ & 1371.50 & 965.00 & 20.00 & 21.94 & 1.15 \\
No noise & 1336.50 & 907.00 & 0.00 & 27.20 & 0.98 \\
\hline
\end{tabular}

\section{Neural envelope tracking and listening effort}

The second aim of this study was to investigate how listening effort and neural envelope tracking are associated. First, we studied the effect of frequency band and speech understanding on neural envelope tracking. Second, we analyzed which listening effort measures contribute to the unexplained variance in neural envelope tracking.

\section{Neural envelope tracking}

To investigate the effect of frequency band on neural envelope tracking, we built an LMM with frequency band as fixed-effect term and participant nested in frequency band as randomeffect. The LMM detected a significant main effect of frequency band. More specifically, lower envelope tracking was observed for theta compared to delta $(\beta=-0.02$, SE $=7.21 \mathrm{e}-$ $03, \mathrm{p}=0.011$, Cohen's $f^{2}(<$ small effect $\left.)=0.0003\right)$. Accordingly, the estimated Bayes factor indicated very strong evidence for $H_{1}$, i.e. frequency band affects neural envelope tracking $\left(B F_{10}(\right.$ Filter $\left.)=73.69\right)$. In addition to the effect of frequency band, adding speech understanding as a fixed-effect term significantly improved the model fit. More specifically, the LMM revealed that envelope tracking was best predicted, i.e. lowest AIC, with speech understanding as a cubic fixed-effect term in addition to frequency band $(\beta=5.51 \mathrm{e}-08$, SE 
$=6.39 \mathrm{e}-09, \mathrm{p}<0.001$, Cohen's $f^{2}$ (large effect) $=0.3987$ ). As shown in figure 5 , envelope tracking increased with speech understanding. This was confirmed by the estimated Bayes factor that revealed extreme evidence for $H_{1}$, indicating that speech understanding modulates neural envelope tracking $\left(B F_{10}(S U)>1000\right.$ with $H_{1}$ : model containing speech understanding and frequency band as fixed-effect terms and $H_{0}$ : model only containing frequency band as fixed-effect term). No significant interaction was observed between speech understanding and frequency band.

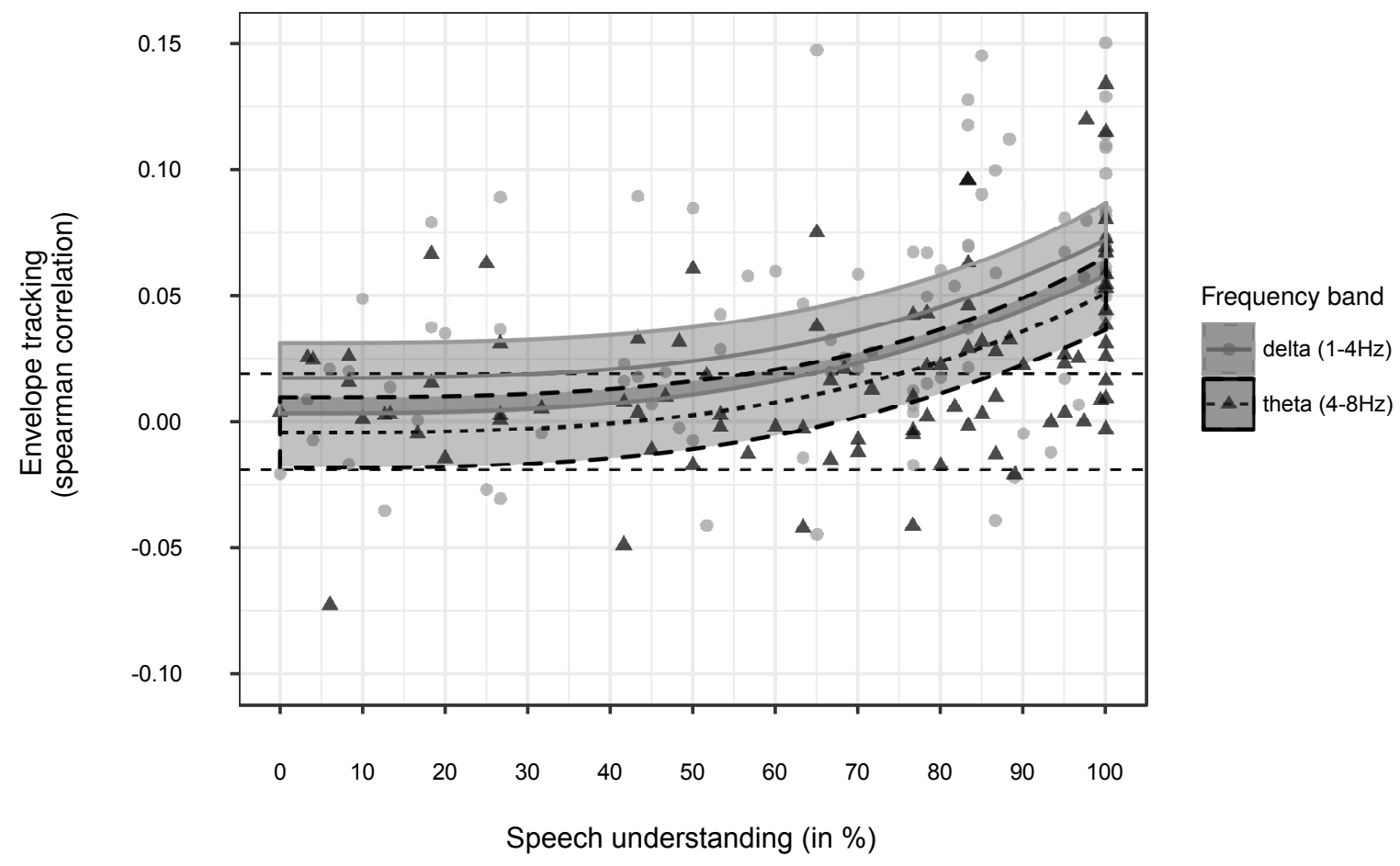

Figure 5: Neural envelope tracking as a function of speech understanding, measured in 13 participants. The regression lines with confidence intervals (shaded areas) indicate that envelope tracking increases supralinearly with increasing speech understanding for both frequency bands. In addition, lower envelope tracking was obtained for theta $(4-8 \mathrm{~Hz})$ versus delta (1-4 Hz) band (color-coded). Dashed black lines indicate the significance level of the measure for envelope tracking.

\section{Link between listening effort and envelope tracking}

We assessed per frequency band whether listening effort accounts for a part of the inter-subject variability in envelope tracking by adding each listening effort measure to the basis model (with $\mathrm{SU}^{3}$ as predictor for envelope tracking). Only for the theta-band (4-8 Hz), adding two 
of the five measures resulted in a significant improvement of the model fit. Likelihood ratio tests and AIC showed no improvement for RTprim, alpha or phase when adding these effort measures as a main or interaction effect with speech understanding to the basis model. For RTsecond and self-report, no improvement was found when adding them as a main effect, but a better model fit was achieved when adding these measures as an interaction with $\mathrm{SU}^{3}$. For both RTsecond and self-report, envelope tracking decreased with increasing effort when participants understood the sentences well whereas envelope tracking increased with increasing effort when it was difficult to understand speech (RTsecond: $\beta=-8.38 \mathrm{e}-08$, SE $=2.91 \mathrm{e}-08, \mathrm{p}=0.005$; self-report: $\beta=-8.15 \mathrm{e}-10, \mathrm{SE}=3.54 \mathrm{e}-10, \mathrm{p}=0.024$; see table 3 ). Although adding these effort measures resulted in a significantly better model fit, the effect size was small when adding RTsecond (Cohen's $f^{2}$ (small effect) $=0.1071$ ) and the estimated Bayes factor revealed only moderate evidence for $H_{1}\left(B F_{10}\left(S U^{3}: R T\right.\right.$ second $)=3.66$ with $H_{1}$ : model with $\mathrm{SU}^{3}$, RTsecond and their interaction as fixed-effect terms and $H_{0}$ : model with only $\mathrm{SU}^{3}$ as fixed-effect term). Similarly, a small effect size was found when adding self-reported effort (Cohen's $f^{2}$ (small effect) $=0.0738$ ) and the estimated Bayes factor only revealed anecdotal evidence for $H_{1}\left(B F_{10}\left(S U^{3}:\right.\right.$ Self - report $)=1.15$ with $H_{1}$ : model with $\mathrm{SU}^{3}$, Self-reported effort and their interaction as fixed-effect terms and $H_{0}$ : model with only $\mathrm{SU}^{3}$ as fixed-effect term).

Table 3: Linear Mixed-effect Models: The effect of listening effort on neural envelope tracking. Regression coefficients ( $\beta$ values), standard errors (SE), degrees of freedom (df), t-Ratios and p-values are reported per fixed-effect term. Participant was included as a random effect.

\begin{tabular}{lllllll}
\hline Measure & Fixed-effect terms & $\beta$ value & SE & df & t-Ratio & p-value \\
\hline \multirow{2}{*}{ RTsecond } & Intercept & $1.27 \mathrm{e}-04$ & $7.53 \mathrm{e}-03$ & 75 & 0.02 & 0.987 \\
& SU $^{3}$ & $5.33 \mathrm{e}-08$ & $1.07 \mathrm{e}-08$ & 75 & 4.98 & $<0.001$ \\
& RTsecond & 0.01 & 0.01 & 75 & 0.96 & 0.342 \\
& RTsecond:SU & $-8.38 \mathrm{e}-08$ & $2.91 \mathrm{e}-08$ & 75 & -2.88 & 0.005 \\
Self-report & Intercept & 0.01 & 0.02 & 75 & 0.62 & 0.540 \\
& SU $^{3}$ & $3.96 \mathrm{e}-08$ & $2.56 \mathrm{e}-08$ & 75 & 1.54 & 0.127 \\
& Self-report & $-7.15 \mathrm{e}-05$ & $2.90 \mathrm{e}-04$ & 75 & -0.25 & 0.806 \\
& Self-report:SU & $-8.15 \mathrm{e}-10$ & $3.54 \mathrm{e}-10$ & 75 & -2.30 & 0.024 \\
\hline
\end{tabular}




\section{Discussion}

The aim of this study was to investigate if listening effort modulates neural envelope tracking. First, we studied the behavior of five effort measures as a function of speech understanding. We found different outcomes among the different measures. Self-report and reaction times on the speech-in-noise test showed a consistent decrease in effort with increasing speech understanding. Alpha power and reaction times on the verbal working memory test, on the other hand, showed maximum effort at the intermediate instead of lowest speech understanding levels. Second, our results revealed a significant increase in neural envelope tracking with increasing speech understanding. Only two of the five effort measures accounted for a small part of the inter-subject variability in theta-band envelope tracking, suggesting that listening effort does not substantially modulate envelope tracking in young, NH listeners.

\section{Different measures reflect different aspects of listening effort}

\section{Behavioral, self-report and neural measures of listening effort}

To compare the five listening effort measures, we analyzed each measure as a function of speech understanding (figure 4). Our results show that the self-report measure and RTprim both quadratically decrease with increasing speech understanding. In other words, highest effort was observed at unfavorable SU-levels whereas lowest effort was obtained when participants understand speech best. This behavior is in line with the findings of previous studies (Gatehouse \& Gordon, 1990; Luts et al., 2010; Zekveld et al., 2010; Houben et al., 2013; Wendt et al., 2014). For example, Rudner et al. (2012) asked hearing impaired participants to rate their listening effort using a visual analog scale, across five SU-levels (between $50 \%$ $\mathrm{SU}$ and $95 \% \mathrm{SU}$ ). Their results showed that perceived effort consistently decreased from the highest to lowest SU-level. Similarly, Pals et al. (2015) found longer RTprim in the most difficult conditions and shortest RTprim in the "No noise" condition.

In contrast to this, we found that RTsecond and alpha power were both best modeled using a cubic term, resulting in a peak-shaped behavior. More specifically, RTsecond was short, i.e. low effort, at the extreme unfavorable or favorable SU-levels whereas RTsecond was long at intermediate SU-levels (40-60\%). These results are in agreement with the findings of dual-task studies (Wu et al., 2016) as well as pupillometry studies (Ohlenforst, Zekveld, \& Lunner et al., 2017) that investigated listening effort across a wide range of SNRs. Similarly, highest alpha power was obtained at intermediate SU-levels (60-80\%). Although increased alpha power has been associated with increased demands or effort (Obleser et al., 2012; 
Dimitrijevic et al., 2019), to our knowledge no research has shown a peak-shaped behavior for alpha power similar to the present study.

In sum, the consistent decrease in effort versus peak-shape indicate that different methods indeed tap into different aspects of listening effort (Lemke \& Besser, 2016; Alhanbali et al., 2019).

\section{Perceived effort versus processing load}

Since self-reported effort and RTprim behave similarly in the present study, we could assume that they reflect the same aspect of listening effort. However, self-report measures are often considered to measure perceived effort whereas single-task paradigm reaction times (RTprim) are assumed to reflect the cognitive processing time required to understand speech (Pals et al., 2015). A possible explanation could be that these measures behave similarly because in our study self-report and RTprim did not measure the motivation of the participant. In the FUEL framework of Pichora-Fuller et al. (2016), listening effort is modeled as a function of both demands as well as the motivation of a person to complete the task. Self-reported effort was measured by the question "How much effort did you have to expend to understand the sentences?". This is somewhat ambiguous, because participants could interpret this question in different ways. Recent studies have suggested that participants tend to substitute effort questions by an easier, perceived performance question (Moore \& Picou, 2018; Picou \& Ricketts, 2018), resulting in highest effort ratings for the most unfavorable SNRs. This could be solved by asking questions without explicitly using the term "effort" (Moore \& Picou, 2018). For example, the question "How hard were you trying to understand the speech" (Wu et al., 2016) or asking to also indicate how often they had given up trying to perceive the sentences (Zekveld et al., 2014), can both give an indication of the aspect motivation and could consequently have resulted in a peak-shaped curve similar to RTsecond (Wu et al., 2016). When examining listening effort at a single SNR, Picou \& Ricketts (2018) advise the use of ratings about the desire to improve the listening condition in order to measure expended effort. Similar to the self-report effort questions, we believe that RTprim can also be biased by the performance on the speech-in-noise test whereas dual-task performance (RTsecond) solely measures the performance on the secondary task. When participants give up, automatically more resources will be available resulting in a better performance on the secondary task. For RTprim on the other hand, poor performance on the speech-in-noise test can result in a longer processing time to decide what to answer.

No consensus is yet established about which listening effort aspect phase synchronization and alpha power represent. Our results have shown no significant effect of speech understanding 
on phase synchronization. There are a few methodological differences with Bernarding et al. (2014) that could explain this. For example, their participants were instructed to recall sentences whereas our participants needed to listen to concatenated sentences. When listening to sentences instead of recalling, attention can drift away more easily resulting in a weaker phase synchronization. This however does not seem a likely reason as we asked questions to keep participants alert. Secondly, Bernarding et al. (2014) varied effort by changing hearing aid settings while we manipulated effort by changing speech understanding. In view of this, several factors seem to affect the sensitivity of phase synchronization to be used as a robust neural correlate of listening effort. Although the estimated Bayes factor only revealed anecdotal evidence for the null hypothesis and the sample size of the present study was relatively small $(\mathrm{N}=13)$, Bernarding et al. (2014) recruited fourteen participants and found similar effects of changing hearing aid settings on phase synchronization and self-reported effort. Hence, our sample size should probably be large enough to detect similar effects of speech understanding on phase synchronization. Nevertheless, we recommend future researchers to collect more data because this will allow them to obtain more conclusive results on this matter. Finally, our results showed a peak-shaped curve for alpha power as a function of speech understanding. Although, no study has yet investigated changes in alpha power across a wide range of SNRs or SU-levels, several studies demonstrated that increased alpha power is observed with increased demand or effort (Obleser et al., 2012; Miles et al., 2017; Dimitrijevic et al., 2019). Nevertheless, based on the estimated Bayes factor (moderate evidence for $H_{1}$ ) and the relatively small effect size, it would be beneficial to conduct new studies with larger sample sizes. Hence, it can be confirmed if alpha power also shows a peak-shaped behavior in other studies and consequently reflects processing load.

\section{Neural envelope tracking and listening effort}

\section{Neural envelope tracking as a function of speech understanding}

We measured neural envelope tracking for two frequency bands. For both the delta- and theta-band, an increase in neural envelope tracking with increasing speech understanding was observed. This is in line with the findings of previous studies in which neural envelope tracking of intelligible sentences or story was measured as a function of SNR (Vanthornhout et al., 2018; Etard \& Reichenbach, 2019; Lesenfants et al., 2019). Hence, our results further confirm the potential of neural envelope tracking as an objective measure of speech understanding (Vanthornhout et al., 2018; Lesenfants et al., 2019). 


\section{Self-report and behavioral effort measures explain a small part of the variability in envelope tracking}

Recent research has demonstrated the influence of several top-down processes on neural envelope tracking (Ding \& Simon, 2012; attention: Das et al., 2016; prior knowledge: Di Liberto et al., 2018). We investigated whether the effort that participants expend when listening to matrix sentences modulates envelope tracking. For the delta-band, no significant association was found between listening effort and neural envelope tracking. These results are in line with a recent study of Müller et al. (2019) who recruited 20 young, NH participants and measured listening effort using self-report and pupillometry, and neural envelope tracking using the cross-correlation between the onset envelope and EEG response. Even though all measures were similarly affected by speech rate, no significant correlations were found between listening effort and neural envelope tracking. According to Müller et al. (2019), this is due to different factors influencing neural envelope tracking versus self-reported effort and pupillometry. In line with this, we suggest the following hypothesis. It is widely known that effortful listening involves attentional processes (Kahneman, 1973; Pichora-Fuller et al., 2016). Based on studies that have shown robust enhanced envelope tracking for selectively attended versus ignored speech (Ding \& Simon, 2012; O'Sullivan et al., 2015; Das et al., 2016), it may be assumed that effort also modulates envelope tracking. However, effortful listening may entail more than selective attention which refers to the process of how the brain neurally segregates different speech streams and selects one of the streams. For example, two individuals may perform equally well in separating speech from noise but one person may have to expend more brain resources or effort, to achieve neural segregation compared to the other person. In view of this, delta-band neural envelope tracking could be more sensitive to the influences of selective attention, i.e. the segregation and selection of acoustic speech streams, compared to changes in listening effort.

For the theta-band, two of our five listening effort measures, RTsecond and self-reported effort, explained a significant, small part of the inter-subject variability of neural envelope tracking. More specifically, when participants understood the sentences well (50-100\%), envelope tracking decreased with increased effort. For difficult speech understanding levels (0-50\%), on the other hand, we observed the opposite, i.e. higher envelope tracking was associated with increased effort. This latter finding is in agreement with the results of Wisniewski et al. (2015) who observed a greater power for frontal midline theta-band activity when participants expend more effort during a speech recognition task. Although our results partly corroborate with the literature, the small effect size, the fact that only two of the five effort measures explained additional variability and Bayes factors revealing only moderate to anecdotal evidence for the alternative hypothesis, could suggest that envelope tracking 
is not substantially modulated by listening effort. This is also supported by the findings of Müller et al. (2019) who recruited twenty participants and did not observe an association between listening effort and envelope tracking. In contrast to this, our retrospective power analysis (simr R package; Green \& Macleod, 2016; Green et al., 2019) indicated that we did not achieve adequate power with thirteen participants to detect these small effects of listening effort on neural envelope tracking, beyond speech understanding (power was lower than 80\%). For the effect of speech understanding on the different effort measures and neural envelope tracking, thirteen participants were sufficient to ensure adequate power. We have to note, however, that post-hoc power analyses are circular and thus provide limited information. In view of all of this, we believe that future studies with larger sample sizes should be conducted in order to further explore and verify to what extent listening effort modulates theta-band neural envelope tracking.

Lastly, the observation of decreased envelope tracking with increased effort for highly intelligible speech understanding levels, was not completely in line with our original hypothesis. Based on previous studies, we expected to find lower envelope tracking for speech without a masker compared to speech presented at a high SNR, due to lower effort. For example, Das et al. (2018) found lower neural envelope tracking for stories presented without a masker compared to stories presented at -1.1 dB SNR. Similarly, Lesenfants et al. (2019) observed lower envelope tracking in the theta-band for matrix sentences presented without a masker compared to sentences presented at $-3.5,-1,-0.5$ or $2.5 \mathrm{~dB}$ SNR. As shown in figure 5 , we found, in contrast to these studies, highest envelope tracking in the "No noise" condition in which $100 \%$ speech understanding was achieved. Therefore, adding our listening effort measures to the basis model for neural envelope tracking could not have resulted in the expected effect. More research is needed to unravel why previous studies have observed lower envelope tracking in young, normal-hearing listeners when they were listening to sentences presented without a masker, compared to equally intelligible sentences, but embedded in background noise.

\section{Future work}

Recently, studies have shown that envelope tracking increases with advancing age. Older NH adults showed an enhanced envelope tracking, which appears to be associated with the age-related deterioration of cognitive processes (Presacco et al., 2016; Decruy et al., 2019). Taking into account that older adults expend more effort during listening (Anderson Gosselin \& Gagné, 2011; Degeest et al., 2015), this enhanced envelope tracking could reflect increased listening effort. Although we have only found a small effect in the present study, including 
effort measures in future aging studies could be beneficial to further investigate this.

In addition to the effect of age, it would be also highly interesting to examine listening effort and neural envelope tracking in hearing impaired (HI) adults. Hearing loss degrades the input signal and consequently creates additional acoustic challenges when listening to speech in noise. Compared to NH adults, HI listeners may expend increased effort to compensate for their hearing loss. A recent review of Ohlenforst, Zekveld, \& Jansma et al. (2017) has pointed out that only physiological measures reveal a consistent increased effort in HI versus NH adults across studies. Furthermore, it would also be valuable to examine which of our effort measures reveals the benefit of aided listening or a particular hearing aid setting at realistic daily-life, highly intelligible SNRs (Rudner et al., 2012; Hornsby, 2013; Bernarding et al., 2014; Picou \& Ricketts, 2018). When examining this, it would be also valuable to investigate which aspect(s) of effort (demands or also motivation) are clinically interesting, which measure is most reliable to capture it (Alhanbali et al., 2019) and which amount of change in effort is meaningful.

Based on our results in young NH participants, we would suggest the use of self-report measures to quantify listening effort as they can be very easily implemented in an existing speech-in-noise test. In addition, studies have shown that self-reported effort can not only reflect perceived effort but also processing load if participants are also instructed to indicate when they give up (Zekveld et al., 2014; Wu et al., 2016). This way, demands as well as motivation, which are suggested to both affect effort, will be measured (Pichora-Fuller et al., 2016; Peelle, 2018). However, we have to note that older adults tend to underestimate their degree of difficulties (Anderson Gosselin \& Gagné, 2011). Because this can affect self-reported

effort, it should be investigated whether alpha power or behavioral effort measures correlate better with the neural envelope tracking in older NH and HI adults.

\section{Conclusion}

In the present study, we investigated listening effort across a wide range of subject-specific SNRs. Our results revealed that different measures indeed lead to different outcomes and support the importance of including a measure of motivation as well as demands to reliably quantify listening effort. With regard to neural envelope tracking, we observed an increase in envelope tracking with increasing speech understanding. Hence, our results provide strong evidence for potential of neural envelope tracking as an objective measure speech understanding in young, NH adults. 


\section{Acknowledgments}

The authors want to thank all participants for their participation in this study as well as research student Maud Lobel for her help in participant recruitment and data acquisition. We also want to acknowledge the section editor and anonymous reviewers for their constructive feedback and help in improving the manuscript.

This work was supported by the Europe project and Research Council (ERC) under the European Union's Horizon 2020 research and innovation programme (Tom Francart; grant agreement No. 637424). Further financial support was provided by the KU Leuven Special Research Fund under grant OT/14/119 to Tom Francart. Research of Jonas Vanthornhout was funded by a PhD grant of the Research Foundation Flanders (FWO).

\section{Conflict of Interest Statement}

The authors declare that there are no potential sources of conflict of interest.

\section{Author Contributions}

L.D., D.L., J.V., T.F. conceived and designed the research; L.D. performed experiments; L.D. analyzed data; L.D., D.L., J.V., T.F. interpreted results of experiments; L.D. prepared figures; L.D, D.L., J.V., T.F. drafted the manuscript. L.D., D.L., J.V., T.F. approved the final version of manuscript.

\section{Abbreviations}

dB SNR, decibel Signal-to-noise ratio; dB HL decibel hearing level; dB SPL, decibel sound pressure level; df, degrees of freedom; EEG, electroencephalography; LMM, Linear Mixedeffect Model; MAD, median absolute deviation; NH, normal-hearing; RTprim, Reaction time on primary task; RTsecond, Reaction time on secondary task; SE, standard error; SNR, signal-to-noise ratio; SRT, speech reception threshold; SU, speech understanding; SWN, speech-weighted noise 


\section{References}

Akaike, H. (1974) A New Look at the Statistical Model Identification. IEEE Transactions on Automatic Control, 19, 716-723.

Alhanbali, S., Dawes, P., Millman, R.E., \& Munro, K.J. (2019) Measures of Listening Effort Are Multidimensional. Ear \& Hearing, 40, 1084-1097.

Anderson Gosselin, P. \& Gagné, J.P. (2010) Use of a Dual-Task Paradigm to Measure Listening Effort. Canadian Journal of Speech-Language Pathology and Audiology/Revue canadienne d'orthophonie et d'audiologie, 34, 43-51.

Anderson Gosselin, P. \& Gagné, J.P. (2011) Older Adults Expend More Listening Effort Than Young Adults recognizing Speech in Noise. Journal of Speech, Language, and Hearing Research, 54, 944-958.

Barton, K. (2019) Multi-Model Inference - Package 'MuMIn'.

Bernarding, C., Strauss, D.J., Hannemann, R., Seidler, H., \& Corona-Strauss, F.I. (2013) Neural correlates of listening effort related factors: Influence of age and hearing impairment. Brain Research Bulletin, 91, 21-30.

Bernarding, C., Strauss, D.J., Hannemann, R., Seidler, H., \& Corona-Strauss, F.I. (2014) Objective assessment of listening effort in the oscillatory EEG: Comparison of different hearing aid configurations. In 2014 36th Annual International Conference of the Ieee Engineering in Medicine and Biology Society, Chicago, Il. IEEE, pp. 2653-2656.

Bertoli, S. \& Bodmer, D. (2016) Effects of age and task difficulty on ERP responses to novel sounds presented during a speech-perception-in-noise test. Clinical Neurophysiology, 127, $360-368$.

Biesmans, W., Das, N., Francart, T., \& Bertrand, A. (2017) Auditory-Inspired Speech Envelope Extraction Methods for Improved EEG-Based Auditory Attention Detection in a Cocktail Party Scenario. IEEE Transactions on Neural Systems and Rehabilitation Engineering, 25, 402-412.

Brand, T. \& Kollmeier, B. (2002) Efficient adaptive procedures for threshold and concurrent slope estimates for psychophysics and speech intelligibility tests. The Journal of the Acoustical Society of America, 111, 2801-2810.

Brus, B.T. \& Voeten, M.J.M. (1973) Een-Minuut-Test. Vorm A en B. Berkhout Testmateriaal, Nijmegen.

Cervantes Constantino, F. \& Simon, J.Z. (2018) Restoration and Efficiency of the Neural 
Processing of Continuous Speech Are Promoted by Prior Knowledge. Frontiers in Systems Neuroscience, 12, 56.

Cohen, J.E. (1988) Statistical Power Analysis for the Behavioral Sciences. Hillsdale, NJ: Lawrence Erlbaum Associates, Inc.

Coren, S. (1993) The lateral preference inventory for measurement of handedness, footedness, eyedness, and earedness: Norms for young adults. Bulletin of the Psychonomic Society, 31, 1-3.

Das, N., Bertrand, A., \& Francart, T. (2018) EEG-based auditory attention detection: Boundary conditions for background noise and speaker positions. Journal of Neural Engineering, 15, 066017.

Das, N., Biesmans, W., Bertrand, A., \& Francart, T. (2016) The effect of head-related filtering and ear-specific decoding bias on auditory attention detection. Journal of Neural Engineering, 13, 056014.

De Vos, A., Vanvooren, S., Vanderauwera, J., Ghesquière, P., \& Wouters, J. (2017) Atypical neural synchronization to speech envelope modulations in dyslexia. Brain and Language, 164, 106-117.

Decruy, L., Vanthornhout, J., \& Francart, T. (2019) Evidence for enhanced neural tracking of the speech envelope underlying age-related speech-in-noise difficulties. Journal of Neurophysiology, 122, 601-615.

Degeest, S., Keppler, H., \& Corthals, P. (2015) The Effect of Age on Listening Effort. Journal of Speech, Language, and Hearing Research, 24, 1-14.

Di Liberto, G.M., Lalor, E.C., \& Millman, R.E. (2018) Causal cortical dynamics of a predictive enhancement of speech intelligibility. NeuroImage, 166, 247-258.

Dimitrijevic, A., Smith, M.L., Kadis, D.S., \& Moore, D.R. (2019) Neural indices of listening effort in noisy environments. Scientific Reports, 9, 1-10.

Ding, N. \& Simon, J.Z. (2012) Emergence of neural encoding of auditory objects while listening to competing speakers. Proceedings of the National Academy of Sciences of the United States of America, 109, 11854-11859.

Ding, N. \& Simon, J.Z. (2014) Cortical entrainment to continuous speech: functional roles and interpretations. Frontiers in Human Neuroscience, 8, 311.

Etard, O. \& Reichenbach, T. (2019) Neural speech tracking in the theta and in the delta frequency band differentially encode clarity and comprehension of speech in noise. Journal of 
Neuroscience, 39, 5750-5759.

Etz, A., Gronau, Q.F., Dablander, F., Edelsbrunner, P.A., \& Baribault, B. (2018) How to become a Bayesian in eight easy steps: An annotated reading list. Psychonomic Bulletin and Review, 25, 219-234.

Field, A., Miles, J., \& Field, Z. (2012) Mixed Designs (GLM 5). In Discovering Statistics Using R. SAGE Publications Inc., pp. 604-652.

Francart, T., Wieringen, A. van, \& Wouters, J. (2008) APEX 3: a multi-purpose test platform for auditory psychophysical experiments. Journal of Neuroscience Methods, 172, 283-293.

Gagné, J.P., Besser, J., \& Lemke, U. (2017) Behavioral assessment of listening effort using a dual-task paradigm: A review. Trends in Hearing, 21, 1-25.

Gatehouse, S. \& Gordon, J. (1990) Response times to speech stimuli as measures of benefit from amplification. British Journal of Audiology, 24, 63-68.

Green, P. \& Macleod, C.J. (2016) SIMR: An R package for power analysis of generalized linear mixed models by simulation. Methods in Ecology and Evolution, 7, 493-498.

Green, P., MacLeod, C., \& Alday, P. (2019) Power Analysis for Generalised Linear Mixed Models by Simulation - Package 'simr'.

Hornsby, B.W.Y. (2013) The effects of hearing aid use on listening effort and mental fatigue associated with sustained speech processing demands. Ear \& Hearing, 34, 523-534.

Houben, R., Van Doorn-Bierman, M., \& Dreschler, W.A. (2013) Using response time to speech as a measure for listening effort. International Journal of Audiology, 52, 753-761.

Jarosz, A.F. \& Wiley, J. (2014) What Are the Odds? A Practical Guide to Computing and Reporting Bayes Factors. The Journal of Problem Solving, 7, 1037-1040.

Jeffreys, H. (1961) Theory of probability (3 ed.). Oxford, UK: Oxford University Press.

Kahneman, D. (1973) Attention and effort. Englewood Cliffs, NJ: Prentice-Hall.

Koelewijn, T., Zekveld, A.A., Festen, J.M., \& Kramer, S.E. (2014) The influence of informational masking on speech perception and pupil response in adults with hearing impairment. The Journal of the Acoustical Society of America, 135, 1596-1606.

Lalor, E.C. \& Foxe, J.J. (2010) Neural responses to uninterrupted natural speech can be extracted with precise temporal resolution. European Journal of Neuroscience, 31, 189-193.

Lalor, E.C., Pearlmutter, B.A., Reilly, R.B., McDarby, G., \& Foxe, J.J. (2006) The VESPA: 
A method for the rapid estimation of a visual evoked potential. NeuroImage, 32, 1549-1561.

Lalor, E.C., Power, A.J., Reilly, R.B., \& Foxe, J.J. (2009) Resolving Precise Temporal Processing Properties of the Auditory System Using Continuous Stimuli. Journal of Neurophysiology, 102, 349-359.

Lemke, U. \& Besser, J. (2016) Cognitive load and listening effort: Concepts and age-related considerations. Ear $\& 3$ Hearing, 37, 77S-84S.

Lesenfants, D., Vanthornhout, J., Verschueren, E., Decruy, L., \& Francart, T. (2019) Predicting individual speech intelligibility from the cortical tracking of acoustic- and phonetic-level speech representations. Hearing Research, 380, 1-9.

Luts, H., Eneman, K., Wouters, J., Schulte, M., Vormann, M., Buechler, M., Dillier, N., Houben, R., Dreschler, W.a., Froehlich, M., Puder, H., Grimm, G., Hohmann, V., Leijon, A., Lombard, A., Mauler, D., \& Spriet, A. (2010) Multicenter evaluation of signal enhancement algorithms for hearing aids. The Journal of the Acoustical Society of America, 127, 1491.

Luts, H., Jansen, S., Dreschler, W., \& Wouters, J. (2014) Development and normative data for the Flemish/Dutch Matrix test 1-6.

Miles, K., McMahon, C., Boisvert, I., Ibrahim, R., Lissa, P. de, Graham, P., \& Lyxell, B. (2017) Objective Assessment of Listening Effort: Coregistration of Pupillometry and EEG. Trends in hearing, 21, 1-13.

Moore, T.M. \& Picou, E.M. (2018) A potential bias in subjective ratings of mental effort. Journal of Speech, Language, and Hearing Research, 61, 2405-2421.

Morey, R.D. (2019) Computation of Bayes Factors for Common Designs - Package 'BayesFactor'. Cran,

Müller, J.A., Wendt, D., Kollmeier, B., Debener, S., \& Brand, T. (2019) Effect of speech rate on neural tracking of speech. Frontiers in Psychology, 10, 449.

Nakagawa, S., Johnson, P.C.D., \& Schielzeth, H. (2017) The coefficient of determination R 2 and intra-class correlation coefficient from generalized linear mixed-effects models revisited and expanded. Journal of the Royal Society Interface, 14, 20170213.

Nasreddine, Z.S., Phillips, N. a, Bedirian, V., Charbonneau, S., Whitehead, V., Collin, I., Cummings, J.L., \& Chertkow, H. (2005) The Montreal Cognitive Assessment, MoCA: A Brief Screening Tool For Mild Cognitive Impairment. Journal of the American Geriatrics Society, 53, 695-699.

Obleser, J., Wostmann, M., Hellbernd, N., Wilsch, A., \& Maess, B. (2012) Adverse Listening 
Conditions and Memory Load Drive a Common Alpha Oscillatory Network. Journal of Neuroscience, 32, 12376-12383.

Ohlenforst, B., Zekveld, A.A., Jansma, E.P., Wang, Y., Naylor, G., Lorens, A., Lunner, T., \& Kramer, S.E. (2017) Effects of Hearing Impairment and Hearing Aid Amplification on Listening Effort. Ear \& Hearing, 38, 267-281.

Ohlenforst, B., Zekveld, A.A., Lunner, T., Wendt, D., Naylor, G., Wang, Y., Versfeld, N.J., \& Kramer, S.E. (2017) Impact of stimulus-related factors and hearing impairment on listening effort as indicated by pupil dilation. Hearing Research, 351, 68-79.

O'Sullivan, J.A., Power, A.J., Mesgarani, N., Rajaram, S., Foxe, J.J., Shinn-Cunningham, B.G., Slaney, M., Shamma, S.A., \& Lalor, E.C. (2015) Attentional Selection in a Cocktail Party Environment Can Be Decoded from Single-Trial EEG. Cerebral Cortex, 25, 1697-1706.

Pals, C., Sarampalis, A., Rijn, H. van, \& Başkent, D. (2015) Validation of a simple responsetime measure of listening effort. The Journal of the Acoustical Society of America, 138, EL187-EL192.

Peelle, J.E. (2018) Listening Effort: How the Cognitive Consequences of Acoustic Challenge Are Reflected in Brain and Behavior. Ear $\mathscr{6}$ Hearing, 39, 204-214.

Pichora-Fuller, M.K., Kramer, S.E., Eckert, M.A., Edwards, B., Hornsby, B.W.Y., Humes, L.E., Lemke, U., Lunner, T., Matthen, M., Mackersie, C.L., Naylor, G., Phillips, N.A., Richter, M., Rudner, M., Sommers, M.S., Tremblay, K.L., \& Wingfield, A. (2016) Hearing Impairment and Cognitive Energy. Ear 83 Hearing, 37, 5S - 27S.

Picou, E.M. \& Ricketts, T.A. (2014) The effect of changing the secondary task in dual-task paradigms for measuring listening effort. Ear \& Hearing, 35, 611-622.

Picou, E.M. \& Ricketts, T.A. (2018) The relationship between speech recognition, behavioural listening effort, and subjective ratings. International Journal of Audiology, 57, 457-467.

Picou, E.M., Ricketts, T.A., and Hornsby, B.W.Y. (2011) Visual Cues and Listenign Effort: Individual Variability. Journal of Speech Language and Hearing Research, 54, 1416-1430.

Pinheiro, J., Bates, D., DebRoy, S., Sarkar, D., \& Team, R.-C. (2017) nlme: Linear and Nonlinear Mixed Effects Models.

Power, A.J., Colling, L.J., Mead, N., Barnes, L., \& Goswami, U. (2016) Neural encoding of the speech envelope by children with developmental dyslexia. Brain and Language, 160, $1-10$.

Presacco, A., Simon, J.Z., \& Anderson, S. (2016) Effect of informational content of noise on 
speech representation in the aging midbrain and cortex. Journal of Neurophysiology, 116, 2356-2367.

Ratcliff, R. (1993) Methods for Dealing With Reaction Time Outliers. Psychological bulletin, 114, 510-532.

Rouder, J.N., Speckman, P.L., Sun, D., Morey, R.D., \& Iverson, G. (2009) Bayesian t tests for accepting and rejecting the null hypothesis. Psychonomic Bulletin and Review, 16, 225-237.

Rönnberg, J., Lunner, T., Zekveld, A., Sörqvist, P., Danielsson, H., Lyxell, B., Dahlström, Ö., Signoret, C., Stenfelt, S., Pichora-Fuller, M.K., \& Rudner, M. (2013) The Ease of Language Understanding (ELU) model: theoretical, empirical, and clinical advances. Frontiers in Systems Neuroscience, 7, 31 .

Rudner, M., Lunner, T., Behrens, T., Thorén, E.S., \& Rönnberg, J. (2012) Working Memory Capacity May Influence Perceived Effort during Aided Speech Recognition in Noise. Journal of the American Academy of Audiology, 23, 577-589.

Selya, A.S., Rose, J.S., Dierker, L.C., Hedeker, D., \& Mermelstein, R.J. (2012) A practical guide to calculating Cohen's f 2, a measure of local effect size, from PROC MIXED. Frontiers in Psychology, 3, 1-6.

Shannon, R.V., Zeng, F.-G., Kamath, V., Wygonski, J., \& Ekelid, M. (1995) Speech Recognition with Primarily Temporal Cues. Science, 270, 303-304.

Somers, B., Francart, T., \& Bertrand, A. (2018) A generic EEG artifact removal algorithm based on the multi-channel Wiener filter. Journal of Neural Engineering, 15, 036007.

Stefan, A.M., Gronau, Q.F., Schönbrodt, F.D., \& Wagenmakers, E.-j. (2019) A Tutorial on Bayes Factor Design Analysis with Informed Priors. Behavior Research Methods, 51, 1042-1058.

Strauss, D.J., Corona-Strauss, F.I., Trenado, C., Bernarding, C., Reith, W., Latzel, M., \& Froehlich, M. (2010) Electrophysiological correlates of listening effort: Neurodynamical modeling and measurement. Cognitive Neurodynamics, 4, 119-131.

Thomson, D.J. (1982) Spectrum Estimation and Harmonic Analysis. Proceedings of the IEEE, 70, 1055-1096.

Van Den Noort, M., Bosch, P., Haverkort, M., \& Hugdahl, K. (2008) A standard computerized version of the reading span test in different languages. European Journal of Psychological Assessment, 24, 35-42.

Van Lier, H., Drinkenburg, W.H.I.M., Van Eeten, Y.J.W., \& Coenen, A.M.L. (2004) Effects 
of diazepam and zolpidem on EEG beta frequencies are behavior-specific in rats. Neuropharmacology, 47, 163-174.

Vanthornhout, J., Decruy, L., Wouters, J., Simon, J.Z., \& Francart, T. (2018) Speech Intelligibility Predicted from Neural Entrainment of the Speech Envelope. Journal of the Association for Research in Otolaryngology, 19, 181.

Vercammen, C., Goossens, T., Wouters, J., \& Wieringen, A. van (2017) How age affects memory task performance in clinically normal hearing persons. Aging, Neuropsychology, and Cognition, 24, 264-280.

Verschueren, E., Somers, B., \& Francart, T. (2019) Neural envelope tracking as a measure of speech understanding in cochlear implant users. Hearing Research, 373, 23-31.

Wendt, D., Brand, T., \& Kollmeier, B. (2014) An eye-tracking paradigm for analyzing the processing time of sentences with different linguistic complexities. PLoS ONE, 9, e100186.

Whelan, R. (2008) Effective analysis of reaction time data. The Psychological Record, 58, $475-482$.

Wilcox, R.R., Granger, D.A., \& Clark, F. (2013) Modern Robust Statistical Methods: Basics with Illustrations Using Psychobiological Data. Universal Journal of Psychology, 1, 21-31.

Winn, M.B., Wendt, D., Koelewijn, T., \& Kuchinsky, S.E. (2018) Best Practices and Advice for Using Pupillometry to Measure Listening Effort : An Introduction for Those Who Want to Get Started. Trends in Hearing, 22, 1-32.

Wisniewski, M.G., Thompson, E.R., Iyer, N., Estepp, J.R., Goder-Reiser, M.N., \& Sullivan, S.C. (2015) Frontal midline $\theta$ power as an index of listening effort. NeuroReport, 26, 94-99. Wu, Y.H., Stangl, E., Zhang, X., Perkins, J., \& Eilers, E. (2016) Psychometric Functions of Dual-Task Paradigms for Measuring Listening Effort. Ear \& Hearing, 37, 660-670.

Zekveld, A.A. \& Kramer, S.E. (2014) Cognitive processing load across a wide range of listening conditions: Insights from pupillometry. Psychophysiology, 51, 277-284.

Zekveld, A.A., Heslenfeld, D.J., Johnsrude, I.S., Versfeld, N.J., \& Kramer, S.E. (2014) The eye as a window to the listening brain: Neural correlates of pupil size as a measure of cognitive listening load. NeuroImage, 101, 76-86.

Zekveld, A.A., Koelewijn, T., \& Kramer, S.E. (2018) The Pupil Dilation Response to Auditory Stimuli : Current State of Knowledge. Trends in Hearing, 22, 1-25.

Zekveld, A.A., Kramer, S.E., \& Festen, J.M. (2010) Pupil Response as an Indication of Effortful Listening: The Influence of Sentence Intelligibility. Ear $\&$ Hearing, 31, 480-490. 